Aging Clin. Exp. Res. 11: 1-3, 1999

\title{
Cell culture aging: Insights for cell aging in vivo?
}

\author{
V.J. Cristofalo \\ Lankenau Medical Research Center, Wynnewood, Pennsylvania; Department of Biochemistry and Molecular \\ Pharmacology, Thomas Jefferson University, Philadelphia, Pennsylvania, U.S.A.
}

The bench mark experiments of Hayflick and Moorhead (1) and Hayflick (2) made it clear that normal human cells in culture display a limited replicative life span.

Careful observation of the deterioration in the replicative ability of the cell population, and the morphological and physiological changes that accompanied the proliferative changes led to the interpretation that this phenomenon was reminiscent of cell aging in situ and, indeed, was human cell aging in culture (3).

Initially, both the observation and the interpretation were controversial. Many in the scientific community, especially gerontologists, resisted the idea that in the presence of adequate nutrition and an otherwise supportive environment, replication could be limited by intrinsic factors, and that the individual cells would undergo senescence in a controlled environment outside the body. However, this finding has been duplicated in many laboratories throughout the world. Furthermore, this general phenomenon of replicative decline during serial subculture was shown to occur in other cell types, such as smooth muscle cells, keratinocytes, endothelial cells and glial cells (reviewed by Cristofalo et al., 4). Gradually through the 1970's, 1980's and 1990's, the validity of the observation of the limited replicated life span of human cells in culture was accepted, although the relationship of the information thus derived to the mechanisms of organismic aging remained controversial.

Among the evidence in support of the relevance of senescence in human cell cultures to aging in situ, the most cited experiments are those showing a small but statistically significant negative correlation between the replicative life span of human skin fibroblast cultures and the age of the donor $(2,5-8)$.

For most investigators, these studies have been the keystone of the arguments for the relevance of cell culture aging to organismic aging.

In a recently published study, we reported the replicative life spans of 124 skin fibroblast cultures, ranging in age from fetal to 94 years (9). Eight of the donors were fetal-derived, while the rest were volunteers in the Baltimore Longitudinal Study on Aging (BLSA). The donors were certified "healthy" at the time of the biopsy by the criteria used in the BLSA. We found high variability in the replicative potential of different cell lines (as others did), but no clear correlation between donor age and fibroblast replicative life span. A similar finding was reported by Goldstein et al. (10) when the donors were carefully screened and excluded if diabetes was present. In addition, Griselli et al. (11) have shown no difference in replicative life span between fibroblast cultures from centenarians and younger individuals.

Cristofalo et al. (9) also compared skin fibroblast cell lines from six individuals who provided skin samples sequentially during their participation in the BLSA, and found no correlation between the age at which the sample was taken and the replicative life span of the corresponding culture.

Unfortunately, these findings, which serve to sharpen our understanding of cell culture senescence, have led some workers to conclude that studies of the aging of cell cultures have no relevance whatsoever to cell aging in the organism. This is unfortunate and misleading. Of course, findings in cell culture are potentially relevant to aging in the organism. The major question, as with any model system, is how the results obtained with cell culture should be interpreted.

In my opinion, the lack of correlation between donor age and replicative life span is of little consequence to the wisdom of using cell cultures to model aging changes that occur in vivo. There is no requirement that a correlation must exist between replicative life span of cell cultures and donor age for the model to be valid and relevant to study the mechanisms of aging changes. Cell cultures have been and are used as heuristic models to examine numerous processes. In fact, much of our knowledge of cancer

(0)1999, Editrice Kurtis

Correspondence: V.J.Cristofalo, Ph.D., The Lankenau Medical Research Center, 100 Lancaster Avenue, Wynnewood, PA 19096, U.S.A. 
biology derives from studies on cell cultures. This is especially true for studies on growth control; and the cells used most frequently, fibroblasts, are not the usual progenitors of tumors in situ.

But the problem is not simple. Aging in vivo is a complex process involving multiple cell types. The organismic aging process is not likely to be duplicated by a single cell type in a defined environment.

Furthermore, for many years we and others have known and puzzled over the fact that the very process of establishing cell cultures and measuring replicative life span selects for the cells with the greatest replicative potential in the tissue sample. Thus, the process itself underestimates in vivo age differences. What's more, the replicative potentials of cells from different biopsies from the same individual are very heterogeneous (6) .

Finally, we know from cell culture studies that replicative potentials reflect replicative history, and not the passage of chronological time (12). Presumably, the multiple fibroblast stem cell beds in vivo would have a heterogeneous replicative history, and would not likely yield homogenous samples and correlations with donor age.

Considering these facts, in retrospect we might have predicted that when donor health and biopsy conditions were controlled, any correlation between donor age and replicative life span for humans would be difficult to demonstrate. This does not mean that a correlation does not exist. For example, in studies with cultures derived from skin biopsies of Fisher 344 rats of different ages, a highly significant negative correlation with replicative life span was shown (13). Of course, these were inbred animals in a constant environment, so presumably some aspects of heterogeneity were reduced. Similar results were found for syrian hamsters (14).

Other studies (15) using a different cell culture paradigm, i.e., clone size distribution, have also shown a negative correlation of proliferative capacity with age. However, this method does not address replicative life span but is an estimate of the proliferative profile of the cells surviving in primary culture. In our studies (9) we also found differences between fetal and adult derived cultures in initial outgrowth but not in replicative life span (14). So taken together, these data implicate a deteriorating proliferative vigor in the aging organism.

Another question is whether the senescence process that occurs in vitro has a direct counterpart in vivo. Do cells exhibiting replicative senescence in vitro achieve the same phenotype in vivo, and if so, do they follow the same pathway in vivo and in vitro? Two possibilities exist: one is that the "pathway" to the senescence phenotype in vitro is the same in vivo but accelerated. The other is that the "pathway" is different and unique to the cell culture environment, and results from either damage, or "missing signals" that would otherwise be present in situ. As a result, the cells might follow a "default" pathway which fits the definition of a senescence process (i.e., deterioration with a loss of homeostasis), but which may or may not occur in vivo. At present, we do not know which is correct. However, Bayreuther et al. (16) have identified cells at different points of their life history in vivo, which show these same phenotypes in vitro.

So then, what is the significance of findings from cell cultures undergoing replicative senescence to organismic aging? What can cell cultures teach us about the biology of aging?

The power of cell cultures lies in the control of the environment, such that the components are defined and the cells to be studied are free of the homeostatic perturbations from other cell types. For example, whether cell culture aging in vitro represents an accelerated in vivo process or a "default" pathway, studies in cell culture which model the cellular changes that we know occur in vivo, such as DNA damage, the effects of reactive oxygen species, attenuation of stress responses, blunting of signal transduction pathways and abnormal or failed regulation of proliferation, can provide enormous insight into the mechanisms by which these processes are controlled in vivo.

The process of change that takes place in human cell cultures during serial subculture fits any definition of biological senescence. There are changes in size, morphology and metabolism that are reminiscent of cell aging in vivo and lead to a decline in functional capacity and homeostasis. In at least some cases the changes are concordant with changes in vivo (17). For example, as mentioned above, there is a substantial literature which shows a decline in proliferative vigor with aging in vivo (18). True, there is currently no direct evidence that the decline in replicative potential might lead to significant functional deficits during aging; yet a model in which we can examine the mechanisms leading to loss of proliferative vigor or loss of proliferative control will certainly provide relevant information to these processes in vivo.

Similarly, there is a literature which shows concordance in vivo and during replicative senescence of those processes involved in tissue matrix remodeling (reviewed in 17). Studies in vitro could provide insights into the mechanisms underlying the regulation of those changes and the effects of those changes on neighboring cells. 
Ultimately, the understanding of how biological processes are regulated and determined in vivo will be largely the result of studies on cell cultures. Human cell cultures have properties which make them ideal for studying the biology of human aging. These properties include a single cell type, a controlled environment, a normal human genome, and a regulated deteriorative process which leads to senescence. Just as we have learned about the cellular and molecular mechanisms underlying the processes of differentiation and neoplasia from cell cultures, so we can learn about the mechanisms of senescence from cell cultures.

There is no doubt that we must ask the right questions, i.e., those questions which relate to changes in vivo and which can be modeled in cell culture. Perhaps, in many cases in the past, the questions asked have not been the right ones. Nonetheless, the cell culture approach, properly formulated, can bring to bear the considerable power of this technology to dissect the cellular and molecular mechanisms underlying aging changes in vivo. To suggest that lack of a direct, one-to-one correlation between the life span of the model and of the organism renders the model invalid is the gerontological equivalent of throwing out the baby with the bath water.

\section{REFERENCES}

1. Hayflick L., Moorhead P.: The serial cultivation of human diploid cell strains. Exp. Cell Res. 25: 585-621, 1961.

2. Hayflick L.: The limited in vitro lifetime of human diploid cell strains. Exp. Cell Res. 37: 614-636, 1965.

3. Hayflick L.: Aging under glass. Exp. Gerontol. 5: 291-303, 1970.

4. Cristofalo V.J., Volker C., Francis M.K., Tresini M.: Age dependent modifications of gene expression in human fibroblasts. Crit. Rev. Eukaryot. Gene Expr. 8: 43-80, 1998.

5. Schneider E.L., Mitsui Y.: The relationship between in vitro cel lular aging and in vivo human age. Proc. Natl. Acad. Sci. USA 73: 3584-3588, 1976.

6. Martin G.M., Sprague C.A., Epstein C.J.: Replicative life-span of cultivated human cells. Effects of donor's age, tissue, and genotype. Lab. Invest. 23: 86-92, 1970.
7. Le Guilly Y., Simon M., Lenoir P., Bourel M.: Long-term culture of human adult liver cells: morphological changes related to in vitro senescence and effect of donor's age on growth potential. Gerontologia (Basel) 19: 303-313, 1973.

8. Bierman E.L.: The effect of donor age on the in vitro life span of cultured human arterial smooth muscle cells. In Vitro 14: 951-955, 1978.

9. Cristofalo V.J., Allen T.G., Pignolo R.J., Martin B.G., Beck J.C.: Relationship between donor age and the replicative lifespan of human cells in culture: A reevaluation. Proc. Natl. Acad. Sci. USA 95: 10614-10619, 1998.

10. Goldstein S., Moerman E.J., Soeldner J.S., Gleason R.E., Barnett D.M.: Chronologic and physiologic age affect replicative life-span of fibroblasts from diabetic, prediabetic, and normal donors. Science 199: 781-782, 1978.

11. Grassilli E., Bellesia E., Salomoni P., Croce M.A., Sikora E., Radziszewska E., Tesco G., Vergelli M., Latorraca S., Barbieri D., Fagiolo U., Santacaterina S., Amaducci L., Riozzo R., Sorbi S., Franceschi C.: c-fos/c-jun expression and AP-1 activation in skin fibroblasts from centenarians. Biochem. Biophys. Res. Commun. 226: 517-523, 1996.

12. Dell'Orco R.T., Mertens G.B., Kruse P.F. Jr.: Doubling potential and calendar time of human diploid cells in culture. Exp. Cell Res. 84: 363-366, 1974.

13. Pignolo R.J., Masoro E.J., Nichols W.W., Bradt C.I., Cristofalo V.J.: Skin fibroblasts from Fischer 344 rats undergo similar changes in replicative life span but not immortalization with caloric restriction of donors. Exp. Cell Res. 201: 16-22, 1992.

14. Bruce S.A., Deamond S.F., Ts'o P.O.P.: In vitro senescence of Syrian hamster mesenchymal cells of fetal to aged adult origin. Inverse relationship between in vivo donor age and in vitro proliferative capacity. Mech. Ageing Dev. 34: 151-173, 1986.

15. Pendergrass W.R., Li Y., Jiang D., Fei R.G., Wolf N.S.: Caloric Restriction: Conservation of cellular replicative capacity in vitro accompanies life span extension in mice. Exp. Cell Res. 217: 309-316, 1995.

16. Bayreuther K., Rodemann H.P., Hommel R., Dittamann K., Albiez M., Francz P.I.: Differentiation of fibroblast stem cells. Proc. Natl. Acad Sci. USA. 85: 5112-5116, 1988.

17. Cristofalo V.J., Volker C., Allen R.G.: Use of the fibroblast model in the study of cellular senescence. In: Barnett Y., Barnett C.R. (Eds.), Aging Methods and Protocols. Human Press, in press (1999).

18. Rubin H.: Cell aging in vivo and in vitro. Mech. Ageing Dev. 98: 1-35, 1997. 